\title{
Erratum to: HIV-Associate Cryptococcal Meningitis: Bridging the Gap Between Developed and Resource-Limited Settings
}

\author{
Mark W. Tenforde ${ }^{1,2} \cdot$ Rae Wake $^{3,4,5} \cdot$ Tshepo Leeme $^{6} \cdot$ Joseph N. Jarvis ${ }^{6,7,8}$
}

Published online: 17 April 2017

(C) Springer International Publishing AG 2017

Erratum to: Curr Clin Micro Rpt (2016) 3:92-102

DOI: 10.1007/s40588-016-0035-5

The original version of this article contained errors in Table 2. The column to the far right contained the incorrect reference numbers. The correct reference numbers are shown below:

The online version of the original article can be found at http://dx.doi.org/ 10.1007/s40588-016-0035-5

Mark W. Tenforde

mark.tenforde@gmail.com

Rae Wake

rmwake@gmail.com

Tshepo Leeme

tbleeme@yahoo.com

Joseph N. Jarvis

drjoejarvis@gmail.com

1 Division of Allergy and Infectious Diseases, University of Washington School of Medicine, Seattle, WA, USA

2 University of Washington Medical Center, 1959 NE Pacific Street, Health Sciences Division \#356423, Seattle, WA 98195, USA
3 Institute of Infection and Immunity, St. George's University of London, London, UK

4 Centre for Opportunistic, Tropical and Hospital Infections, National Institute for Communicable Diseases, Johannesburg, South Africa

5 National Institute for Communicable Diseases, 1 Modderfontein Road, Sandringham, Johannesburg 2131, South Africa

6 Botswana-UPenn Partnership, Gaborone, Botswana

7 Division of Infectious Diseases, Department of Medicine, Perelman School of Medicine, University of Pennsylvania, Philadelphia, PA, USA

8 Department of Clinical Research, Faculty of Infectious Diseases and Tropical Medicine, London School of Hygiene and Tropical Medicine, London, UK 
Table 2 Recent studies with early mortality estimates for cryptococcal meningitis in resource-limited settings

\begin{tabular}{|c|c|c|c|c|c|c|}
\hline Country & Induction regimen $^{\mathrm{a}}$ & Mortality, \% (n/N) & $\begin{array}{l}\text { Clinical } \\
\text { trial }\end{array}$ & Notes & Year & Ref \\
\hline Botswana & AmB $0.7 \mathrm{mg} / \mathrm{kg}$ daily & $26 \%(7 / 27)$ at 24 weeks & Yes & $\begin{array}{l}\text { Lower mortality in group that } \\
\text { delayed ART for } 28 \text { days } \\
\text { post-randomization }(15 \%[2 / 13]) \\
\text { versus group that initiated ART } \\
\text { within } 7 \text { days of randomization } \\
(36 \%[5 / 14])^{\mathrm{b}}\end{array}$ & 2013 & [42] \\
\hline Cameroon & Regimen not specified & $52 \%(39 / 75)$ died in hospital & No & & 2013 & {$[32]$} \\
\hline Ethiopia & Mixture of $\mathrm{AmB}(n=45)$ and FLU $(n=33)$ & $48 \%$ (37/77) died in hospital & No & & 2012 & [33] \\
\hline Malawi & FLU $1200 \mathrm{mg}$ daily & $55 \%(26 / 47)$ at 10 weeks & Yes & & 2014 & {$[21]$} \\
\hline Malawi & FLU 800 mg daily & $58 \%(35 / 60)$ at 10 weeks & Yes & & 2013 & [20] \\
\hline Malawi & $\begin{array}{l}\text { AmB } 1 \mathrm{mg} / \mathrm{kg} \text { daily for } 7 \text { days }+ \text { FLU } \\
1200 \mathrm{mg} \text { daily; AmB } 1 \mathrm{mg} / \mathrm{kg} \text { daily for } \\
7 \text { days }+5 \mathrm{FC} 100 \mathrm{mg} / \mathrm{kg} \text { daily }+ \text { FLU } \\
1200 \mathrm{mg} \text { daily }\end{array}$ & $33 \%(13 / 39)$ at 10 weeks $^{\mathrm{b}}$ & Yes & $\begin{array}{l}\text { Greater early fungicidal activity with } \\
\text { 3-drug regimen }\end{array}$ & 2012 & [28] \\
\hline Malawi & $\begin{array}{l}\text { FLU } 1200 \mathrm{mg} \text { daily; FLU } 1200 \mathrm{mg} \text { daily } \\
\quad+5 \mathrm{FC} 100 \mathrm{mg} / \mathrm{kg} \text { daily }\end{array}$ & $50 \%(20 / 40)$ at 10 weeks $^{\mathrm{b}}$ & Yes & $\begin{array}{l}\text { Greater early fungicidal activity in } \\
\text { combination regimen }\end{array}$ & 2010 & [23] \\
\hline South Africa & $\begin{array}{l}\text { Pooled patient population from clinical trials } \\
\text { using AmB-based regimen }\end{array}$ & $41 \%(108 / 263)$ at 1 year & Yes & & 2014 & {$[31]$} \\
\hline $\begin{array}{l}\text { South Africa } \\
\text { and Uganda }\end{array}$ & $\begin{array}{l}\text { AmB } 0.7-1 \mathrm{mg} / \mathrm{kg} \text { daily + FLU } 800 \mathrm{mg} \\
\text { daily }\end{array}$ & $38 \%(67 / 177)$ at 26 weeks & Yes & $\begin{array}{l}\text { Lower mortality in group that } \\
\text { delayed ART for } 5 \text { weeks }(30 \% \\
\text { [27/89]) versus group that started } \\
\text { ART 1-2 weeks after diagnosis } \\
(45 \% \text { [40/88]) }\end{array}$ & 2014 & [43] \\
\hline South Africa & $\begin{array}{c}\text { AmB } 0.7-1 \mathrm{mg} / \mathrm{kg} \text { daily }+5 \mathrm{FC} 100 \mathrm{mg} / \mathrm{kg} \\
\text { daily; AmB } 0.7-1 \mathrm{mg} / \mathrm{kg} \text { daily }+\mathrm{FLU} \\
800 \mathrm{mg} \text { daily; AmB } 0.7-1 \mathrm{mg} / \mathrm{kg} \text { daily }+ \\
\text { FLU } 600 \mathrm{mg} \text { daily; AmB } 0.7-1 \mathrm{mg} / \mathrm{kg} \\
\text { daily + voriconazole } 300 \mathrm{mg} \text { twice daily }\end{array}$ & $29 \%(22 / 75)$ at 10 weeks ${ }^{b}$ & Yes & $\begin{array}{l}\text { No difference in early fungicidal } \\
\text { activity between treatment groups }\end{array}$ & 2012 & {$[26]$} \\
\hline South Africa & Regimen not specified & $89 \%(66 / 74)$ at 2 years & No & & 2011 & [35] \\
\hline South Africa & $\begin{array}{l}\mathrm{AmB} 0.7 \mathrm{mg} / \mathrm{kg} \text { daily }+5 \mathrm{FC} 100 \mathrm{mg} / \mathrm{kg} \\
\text { daily; } \mathrm{AmB} 1 \mathrm{mg} / \mathrm{kg} \text { daily }+5 \mathrm{FC} \\
100 \mathrm{mg} / \mathrm{kg} \text { daily }\end{array}$ & $24 \%(15 / 63)$ at 10 weeks $^{\mathrm{b}}$ & Yes & $\begin{array}{l}\text { Greater early fungicidal activity in } \\
\text { group receiving higher dose of } \\
\text { AmB }\end{array}$ & 2008 & [74] \\
\hline South Africa & $\begin{array}{l}\text { AmB } 1 \mathrm{mg} / \mathrm{kg} \text { daily for } 7 \text { days followed by } \\
\text { FLU } 400 \mathrm{mg} \text { daily }\end{array}$ & $33 \%(16 / 48)$ at 10 weeks & No & & 2007 & {$[25]$} \\
\hline Thailand & $\begin{array}{l}\text { AmB } 0.7 \mathrm{mg} / \mathrm{kg} \text { daily; AmB } 0.7 \mathrm{mg} / \mathrm{kg} \\
\text { daily }+5 \mathrm{FC} 100 \mathrm{mg} / \mathrm{kg} \text { daily; AmB } \\
0.7 \mathrm{mg} / \mathrm{kg} \text { daily + FLU } 400 \mathrm{mg} \text { daily; } \\
\text { AmB } 0.7 \mathrm{mg} / \mathrm{kg} \text { daily }+5 \mathrm{FC} 100 \mathrm{mg} / \mathrm{kg} \\
\text { daily + FLU } 400 \mathrm{mg} \text { daily }\end{array}$ & $22 \%(14 / 63)$ at 10 weeks $^{\mathrm{b}}$ & Yes & $\begin{array}{l}\text { Greater early fungicidal activity with } \\
\text { combination } \mathrm{AmB}+5 \mathrm{FC} \text { than } \\
\text { other groups }\end{array}$ & 2004 & {$[24]$} \\
\hline Uganda & $\begin{array}{l}\text { AmB } 1 \mathrm{mg} / \mathrm{kg} \text { daily for } 5 \text { days }+ \text { FLU } \\
1200 \mathrm{mg} \text { daily }\end{array}$ & $28 \%(8 / 29)$ at 10 weeks & Yes & & 2012 & [27] \\
\hline Uganda & AmB $0.7-1 \mathrm{mg} / \mathrm{kg}$ daily & $54 \%(102 / 182)$ at 1 year & No & & 2012 & {$[30]$} \\
\hline Uganda & FLU 800 mg daily; FLU 1200 mg daily & $54 \%(31 / 57)$ at 10 weeks $^{\text {b }}$ & Yes & $\begin{array}{l}\text { Greater early fungicidal activity in } \\
\text { group receiving higher dose of } \\
\text { FLU }\end{array}$ & 2008 & [22] \\
\hline Uganda & AmB $0.7 \mathrm{mg} / \mathrm{kg}$ daily & $59 \%(26 / 44)$ at 6 months & No & & 2008 & [34] \\
\hline Vietnam & $\begin{array}{l}\text { AmB } 1 \mathrm{mg} / \mathrm{kg} \text { daily for } 4 \text { weeks; AmB } \\
1 \mathrm{mg} / \mathrm{kg} \text { daily for } 2 \text { weeks }+5 \mathrm{FC} \\
100 \mathrm{mg} / \mathrm{kg} \text { daily; AmB } 1 \mathrm{mg} / \mathrm{kg} \text { daily for } \\
2 \text { weeks + fluconazole } 400 \mathrm{mg} \text { daily }\end{array}$ & $36 \%(107 / 298)$ at 10 weeks & Yes & $\begin{array}{l}\text { Mortality lower in combined AmB + } \\
\text { 5FC group (30\% [30/100]) } \\
\text { compared to AmB alone (44\% } \\
\text { [44/99]) }\end{array}$ & 2013 & [29] \\
\hline Zimbabwe & Fluconazole $800 \mathrm{mg}$ daily & $73 \%(35 / 48)$ at 3 years & Yes & $\begin{array}{l}\text { Lower mortality in group that } \\
\text { delayed ART for } 10 \text { weeks after } \\
\text { diagnosis }(54 \% \text { [12/22]) versus } \\
\text { group that started ART within } 72 \mathrm{~h} \\
\text { of diagnosis }(88 \% \text { [23/26]) }\end{array}$ & 2010 & [92] \\
\hline
\end{tabular}

$5 F C$ flucytosine, $A m B$ amphotericin B deoxycholate, $A R T$ antiretroviral therapy, $F L U$ fluconazole

${ }^{\mathrm{a}}$ Induction therapy for 14 days unless otherwise noted

${ }^{\mathrm{b}}$ No statistically significant difference between groups 